\title{
Genomic imbalances associated with secondary acute leukemias in Hodgkin lymphoma
}

\author{
GIANLUCA BRUSA ${ }^{1}$, ELISA ZUFFA ${ }^{1}$, CLAUDIA MARIA HATTINGER ${ }^{2}$, MASSIMO SERRA $^{2}$, \\ DANIEL REMONDINI ${ }^{2,6}$, GASTONE CASTELLANI $^{3}$, SIMONA RIGHI $^{4}$, CRISTINA CAMPIDELLI $^{4}$, \\ STEFANO PILERI ${ }^{4}$, PIER LUIGI ZINZANI ${ }^{5}$, ANNALISA GABRIELE ${ }^{5}$, MANUELA MANCINI $^{5}$, \\ PATRIZIA CORRADO ${ }^{5}$, ENZA BARBIERI ${ }^{1}$ and MARIA ALESSANDRA SANTUCCI ${ }^{5}$
}

\author{
${ }^{1}$ Istituto di Radioterapia 'Luigi Galvani', Università di Bologna, Via Massarenti 9, 40138 Bologna; \\ ${ }^{2}$ Laboratorio di Ricerca Oncologica, Istituti Ortopedici Rizzoli, Via di Barbiano 1/10, 40136 Bologna; \\ ${ }^{3}$ Dipartimento di Morfofisiologia Veterinaria e Produzione Animale, Università di Bologna, Ozzano Emilia, \\ 40064 Bologna; ${ }^{4}$ Unità di Emolinfopatologia e ${ }^{5}$ Laboratorio di Biologia Molecolare e Cellulare, Istituto di Ematologia \\ e Oncologia Medica 'Lorenzo e Ariosto Seràgnoli', Università di Bologna, Via Massarenti 9, 40138 Bologna; \\ ${ }^{6}$ Dipartimento di Fisica, Università di Bologna, Viale Berti-Pichat 6/2, 40136 Bologna, Italy
}

Received June 26, 2007; Accepted August 8, 2007

\begin{abstract}
Secondary tumors and leukemias are major complications in Hodgkin lymphoma (HL). They likely arise from clonal selection of cells that have accumulated genomic lesions induced by chemo- and radiotherapy and may be further promoted by the loss of DNA repair and/or other pathways ensuring the fidelity of replicated DNA. To distinguish genomic imbalances associated with the development of acute myeloid leukemia (AML) in HL we used an array-based comparative genomic hybridization (aCGH) strategy on whole lymph node biopsies of HL patient. Genomic imbalances (amplifications and deletions) associated with AML outcome in 3 classic HL patients, at clinical diagnosis they exhibited a discrete individual variability. Three amplifications and 5 deletions were shared by all 3 patients. They involved AFM137XA11, a 9p11.2 pericentric region; FGFR1, the FGF receptor most frequently translocated in AML; PPARBP, a co-activator of nuclear receptors RAR $\alpha$, RXR and TRß1; AFM217YD10, a 17q25 telomeric region; FGR, an SRC2 kinase involved in cytokine production by $\mathrm{NK}$ and $\mathrm{CD} 4{ }^{+} \mathrm{NKT}$ cells; GATA3, a Th2specific transcription factor; TOP1, involved in DNA recombination and repair; WT1, a transcription factor involved in $\mathrm{CD}^{+} \mathrm{T}$ cell response against leukaemic blasts. Immunohistochemistry confirmed aCGH results and distinguished the distribution of either amplified or deleted
\end{abstract}

Correspondence to: Dr Gianluca Brusa, Istituto di Ematologia e Oncologia Medica 'Lorenzo e Ariosto Seràgnoli', Università di Bologna - Medical School, Via Massarenti 9, 40138 Bologna, Italy E-mail: gbrusa@alma.unibo.it

Key words: array-based comparative genomic hybridization, genomic instability, Hodgkin lymphoma, secondary acute leukemias gene products in neoplastic Reed Sternberg (RS) cells and nonneoplastic lymph node components.

\section{Introduction}

Secondary malignancies are major treatment-related complications in HL and a leading cause of death of long-term survivors. They may arise from clonal selection of cells that have accumulated transforming genomic lesions induced by chemo- and radiotherapy. DNA repair defects are assumed to increase the susceptibility to treatment-related cancers (1).

Hodgkin and Reed-Sternberg (H/RS) cells, the pathognomonic cells of HL, are aberrant post-germinal center B cells that have lost their identity as a consequence of the abolition of $\mathrm{B}$ cell gene transcription program and evade apoptotic cell death through CD30 signaling, NF- $\mathrm{B}$ activation and Fas pathway failure (2). Virtually all $\mathrm{H} / \mathrm{RS}$ cells exhibit neither clonal nor disease-specific chromosomal abnormalities that may originate from their own or their progenitor inherent genomic instability (3). The hypothesis of an unstable genetic background concurrently promoting the evasion of H/RS cell progenitors from FAS-mediated negative selection in the germinal centre, accumulation of multiple genetic aberrations driving their progression towards a fully transformed phenotype and deregulated differentiation culminating in endomitosis is advanced by the findings that chromosomal abnormalities (eventually resulting in oncosuppressor loss) are not restricted to the malignant cellular context (4-8). The association of polymorphisms in DNA repair and oxidative stress response genes with an increased risk for non-Hodgkin lymphoma supports a role of individual genetic susceptibility in primary B lymphomagenesis $(9,10)$. However, the determining factors of chromosomal lesions in HL are still elusive. Most likely, they do not include defects of nucleotide mismatch repair resulting in microsatellite instability, a putative source of genomic instability in H/RS cells (11). 
Genomic instability in therapy-related myelodysplastic syndromes and AMLs seems genetically determined. It might stem from defects of detoxification systems designed to limit oxidative DNA damage (driven by polymorphisms of genes involved in drug metabolism) and/or chromatin assembly factors, resulting in accumulation of double strand breaks (DSB) and high levels of sister chromatid exchange (SCE) $(12,13)$. More recently, combined polymorphisms in human homeobox HLX1 (a determinant of hematopoietic stem cell frequency) and DNA repair RAD1 genes have been associated with an increased risk of therapy-related AML (14).

We used an aCGH strategy to detect and map genomic imbalances (amplifications and deletions) in whole formalinfixed, paraffin-embedded lymph nodes of 3 patients with classic HL at diagnosis who developed secondary AML during the follow-up. Our results underscored 3 amplifications and 5 deletions common to all 3 patients. Further investigation is required to elucidate whether those genomic imbalances may be considered as molecular risk factors for secondary leukemogenesis.

\section{Materials and methods}

ACGH analysis. DNA extraction was performed using a commercial kit (QIAamp DNA kit from Qiagen) according to manifacturer's instructions and quantified by means of NanoDrop Technology (Wilmington). Low DNA amounts were amplified by means of DOP-PCR (Roche). GenoSensor array 300 microarrays (Abbott Vysis Inc.) consisted of 287 probes spotted in triplicate. Briefly, test and reference DNAs, after random priming labelling with Cy3-dCTP and Cy5-dCTP (Amersham Biosciences) and electrophoresis resolution in $1.5 \%$ agarose gel to confirm their length range between 100 and $600 \mathrm{bp}$, were denatured at $80^{\circ} \mathrm{C}$ for $10 \mathrm{~min}$ in $25 \mu 1$ hybridization buffer containing human Cot-I DNA and hybridized on microchips at $37^{\circ} \mathrm{C}$ for $72 \mathrm{~h}$. After washing microarrays were counterstained with DAPI IV (Abbott Vysis Inc.). The images were captured and analyzed with the GenoSensor reader system (Abbott Vysis Inc.). Only those probes with at least 2 available spots were considered for analysis (spot measurements were highly reproducible, thus two spots are considered sufficient) and the average over the spots was taken as the copy number ratio for that gene. Thresholds for gene amplification and deletion were 1.2 and 0.82 , respectively (15).

Fluorescence in situ hybridization (FISH). FISH was performed on sections from formalin-fixed, paraffin-embedded biopsy blocks using the following commercially available fluorescence-labelled, locus-specific (LSI) N-myc and CDKN 2A (p16) and associated centromere DNA probes (CEP) (Abbott Vysis Inc.). Fifty nuclei per sample were scored for each LSI probe. Digital images were acquired with a Nikon Eclipse 1000 fluorescence microscope. Amplifications and deletions were detected when the ratio between the number of spots of test gene and of internal reference gene was greater than $2 \mathrm{~N}+1$ or lower than 1 respectively, with $\mathrm{N}$ corresponding to the number of spots of the internal reference gene.

Immunohistochemistry. Tissue sections $(3 \mu \mathrm{m})$ formalinfixed, paraffin-embedded blocks were coated on electrically charged slides, dewaxed and rehydrated, and then submitted to antigen retrieval by micro-waving in $1 \mathrm{mM}$ EDTA ( $\mathrm{pH}$ 8.0) at $900 \mathrm{~W}$ (3 cycles of $5 \mathrm{~min}$ each). After cooling, slides were incubated for $30 \mathrm{~min}$ at room temperature with primary antibodies [PPARBP and GATA3 from Santa Cruz Biotech., Top1, FGFR1 and FGR(SRC2) from Abcam, FES from Abgent, HRAS and WT1 from Dako]. Antibody binding was detected by the alkaline phosphatase anti-alkaline phosphatase technique or by the peroxidase-based LSAB method (Dako) and sections were then counterstained with hematoxylin.

Polymerase chain reaction $(P C R)$. PCR reactions were performed in $\mathrm{T}$ Personal instrument by Biometra according to the following cycle profile: denaturation at $98^{\circ} \mathrm{C}$ for $30 \mathrm{sec}$, annealing at $59^{\circ} \mathrm{C}$ for $30 \mathrm{sec}$ and extension $72^{\circ} \mathrm{C}$ for $1 \mathrm{~min}$. Thirty cycles were carried out for PCR amplification of $\beta$ actin. Amplification products (226 bp) were then resolved in $1 \%$ agar and signal intensities measured by densitometric analysis. 3 -actin primers sequences are: 5'CATGTGCAAG GCCGGCTTC 3' (upper) and 3'GAAGGTGTGTGGTGC CAGATTT 5' (lower). PCR reactions were carried out on a total amount of $500 \mathrm{ng}$ of DOP-PCR (Roche kit) products in PCR reaction buffer containing $50 \mathrm{nM}$ Tris $\mathrm{pH} 8.4,250 \mu \mathrm{g} /$ $\mathrm{ml}$ BSA, $3 \mathrm{mM} \mathrm{MgCl}_{2}, 2 \mathrm{mM}$ dNTPs, $4 \mathrm{U}$ Taq polymerase and $0.5 \mu \mathrm{M}$ specific primers.

\section{Results and Discussion}

The cumulative incidence of secondary acute myeloid leukemias (AML) in HL ranges from $0.8 \%$ to $6.3 \%$. It is mostly restricted to the first 5-7 years after treatment and correlated with the genotoxic damage induced by chemotherapy protocols including alkylating drugs (mechlorethamine, in particular) (12). In our cohort of 1,224 classic HL patients (corresponding to $0.89 \%$ ) 11 underwent this therapy-related outcome (data not shown). To investigate whether individual discrete genomic imbalances at diagnosis would distinguish patients prone to AML development during HL follow-up we used an aCGH strategy (GenoSensor array 300 purchased from Abbott Vysis Inc.). In preliminary experiments (data not shown) we did not detect differences in DNA sequence copy numbers of whole lymph node and peripheral blood mono-nuclear cells from 2 normal persons and 2 classic HL patients at diagnosis (with $<2 \%$ neoplastic component), supporting that aCGH mirror the individual genotypic profiles. However, the putative impact of neoplastic lymph node microenvironment and/or infection (in particular by Epstein-Barr virus) on genomic profiles of diverse lymph node cellular components possibly relevant for HL prognosis requires further investigation in a larger number of patients and using different techniques (aCGH technique only detects amplifications and deletions but does not provide any information on gene expression) $(16,17)$.

Good quality DNA suitable for aCGH analysis was obtained from lymph node biopsies of 3 out of 11 classic HL cases who developed AML (see Table I for clinical details). They were used as tests in a first aCGH series set using pooled DNAs from 8 reactive lymph node biopsies as reference. To confirm the good quality of DNA used in aCGH, PCR reactions for $B$-actin were performed on DOP-PCR products of all patients involved in the study ( 3 classic HL and 3 
Table I. Clinical details of classic HL patients included in the study.

\begin{tabular}{|c|c|c|c|}
\hline & Patient 1 & Patient 2 & Patient 3 \\
\hline \multicolumn{4}{|l|}{ HL } \\
\hline Histological type & $\begin{array}{l}\text { Nodular } \\
\text { sclerosis }\end{array}$ & Unclassified & $\begin{array}{l}\text { Nodular } \\
\text { sclerosis }\end{array}$ \\
\hline $\begin{array}{l}\text { Clinical stage at } \\
\text { diagnosis }\end{array}$ & IV B (bone) & III B & $\begin{array}{c}\text { II A (bulky } \\
\text { mediastinum) }\end{array}$ \\
\hline Chemotherapy & $\begin{array}{l}8 \text { MOPP cycles }+ \\
8 \text { ABVD cycles }\end{array}$ & $\begin{array}{l}6 \text { VBM cycles }+ \\
3 \text { MOPP cycles }\end{array}$ & 6 ABVD cycles \\
\hline Radiotherapy & Not done & $\begin{array}{l}\text { Lomboaortic } \\
\text { lymph nodes, } \\
\text { spleen }\end{array}$ & Not done \\
\hline $\begin{array}{l}\text { Interval between } \\
\text { HL diagnosis and } \\
\text { AML outcome }\end{array}$ & 58 months & 76 months & 25 months \\
\hline AML & & & \\
\hline $\begin{array}{l}\text { Morphologic } \\
\text { subtype }^{\mathrm{a}}\end{array}$ & M1 & M0-M1 & M3 \\
\hline Cytogenetics & $\begin{array}{c}\text { Normal } \\
\text { karyotype }\end{array}$ & $\begin{array}{c}\text { Normal } \\
\text { karyotype }\end{array}$ & $\begin{array}{c}\operatorname{tt}(15 ; 17)(\mathrm{q} 22 ; \mathrm{q} 12) \\
\operatorname{del}(7)(\mathrm{q} 22 ; \mathrm{q} 32) \\
\operatorname{del}(9)(\mathrm{q} 13 ; \mathrm{q} 31)\end{array}$ \\
\hline
\end{tabular}

B, Patients who did not develop AML

\begin{tabular}{lccc}
\hline & Patient 4 & Patient 5 & Patient 6 \\
\hline HL & Nodular & Nodular & Nodular \\
sclerosis & sclerosis & sclerosis \\
Clinical stage at & III A & IV B (lung and & II A (bulky \\
diagnosis & & spleen) & mediastinum) \\
Chemotherapy & 8 MOPP cycles+ & 6 ABVD cycles+ & 4 ABVD cycles \\
& 8 ABVD cycles & laterocervical & laterocervical \\
Radiotherapy & Not done & Sovraclavear and & Sovraclavear and \\
& & lymph nodes, & lymph nodes, \\
& & mediastinum & mediastinum \\
\hline
\end{tabular}

Patients 1, 2 and 3 developed AML. Patients 4,5 and 6, who did not develop any secondary malignancy, were comparable to the first group of patients for

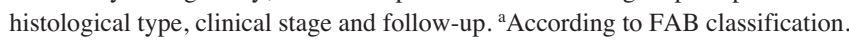

classic HL who developed AML) and of pooled DNAs from normal persons (Fig. 1). Genomic imbalances included 44 amplifications and 28 deletions. Of 44 amplifications, 24 were common to all 3 patients, 17 to 2 patients and the remaining 3 were seen only in 1 patient (Table IIA). Out of 28 deletions, 5 were common to all 3 patients, 12 were shared by 2 patients and the remaining 11 were seen only in 1 patient (Table IIB).

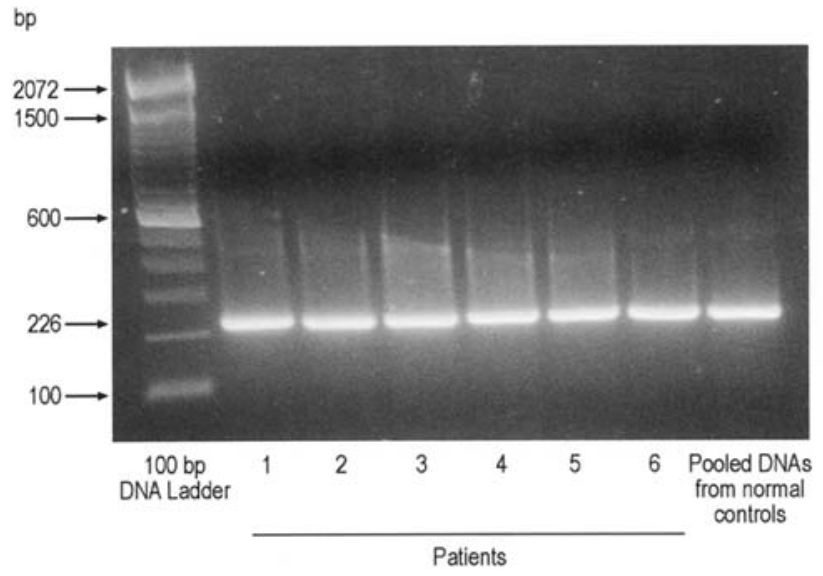

Figure 1. Polymerase chain reaction (PCR) for $\beta$-actin. PCR reactions for $\beta$-actin were performed in DOP-PCR products of all patients involved in the study. Pooled DNAs from normal persons and the six patients showed comparable levels of $\beta$-actin gene, confirming the good quality of DNA used in aCGH.
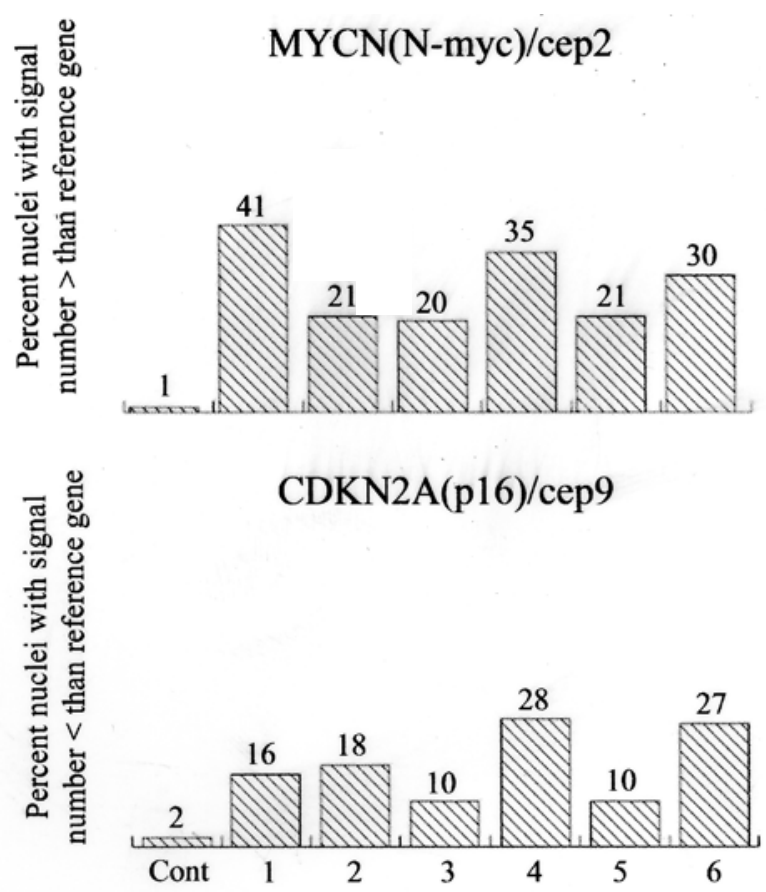

Figure 2. Results of FISH analysis relative to MYCN (N-Myc) and CDKN 2A (p16). FISH analysis was performed on paraffin-embedded lymph node biopsies of normal persons (the only one shown here is representative of all), 3 HL patients who developed AML (bars 1, 2 and 3) and 3 HL patients (comparable for histological type, clinical stage and follow-up) who did not develop any secondary malignancy (bars 4, 5 and 6). FISH was performed on sections from formalin-fixed, paraffin-embedded biopsies as described in Materials and methods. Cep2 and cep 9 centromeric regions were used as references for the signal copy number in single nuclei.

ACGHs results relative to MYCN (N-Myc) amplification and CDKN 2A (p16) deletion were validated by FISH. We analysed ratio between number of spots of test genes [MYCN (N-Myc) and CDKN 2A (p16)] and of internal reference genes (cep2 and cep9 respectively) (Fig. 2). Notably, most genomic imbalances shared by at least 2 classic HL patients who developed AML were also found in aCGH set using DNAs 
Table II. Genomic imbalances in classic HL patients who developed secondary AML relative to normal controls.

A, Amplifications

\begin{tabular}{|c|c|c|c|c|}
\hline $\begin{array}{l}\text { Genes and } \\
\text { regions }\end{array}$ & $\begin{array}{c}\text { Chromosomal } \\
\text { locus }\end{array}$ & 1 & 2 & 3 \\
\hline 16PTEL03 & $16 \mathrm{p}$ tel & 1.22 & 1.42 & 1.66 \\
\hline 20QTEL14 & $20 q$ tel & 1.68 & 1.39 & 2.08 \\
\hline 6QTEL54 & $6 \mathrm{q}$ tel & 1.80 & 1.32 & 2.64 \\
\hline 7QTEL20 & $7 \mathrm{q}$ tel & 1.29 & 1.43 & 2.04 \\
\hline 8M16/SP6 & $12 \mathrm{p}$ tel & 1.50 & 1.46 & 2.68 \\
\hline ARHGAP8 & $22 q 13.3$ & 1.33 & 1.26 & 1.33 \\
\hline ATM & $11 \mathrm{q} 22.3$ & 1.24 & 1.26 & 2.12 \\
\hline CDC2L1(p58) & $1 \mathrm{p} 36$ & 1.21 & 1.41 & 2.18 \\
\hline D10S167 & 10p11-10q11 & 1.26 & 1.26 & 2.03 \\
\hline D13S25 & $13 q 14.3$ & 1.24 & 1.21 & 1.71 \\
\hline D17S1670 & $17 q 23$ & 1.24 & 1.30 & 1.59 \\
\hline EGR1 & $5 q 31.1$ & 1.57 & 1.46 & 1.99 \\
\hline ELN & $7 q 11.23$ & 1.47 & 1.59 & 2.46 \\
\hline ERBB2 (HER-2) & 17q11.2-17q12 & 1.59 & 1.28 & 1.71 \\
\hline FES & $15 q 26.1$ & 1.30 & 1.47 & 1.83 \\
\hline GLI & $12 q 13.2-q 13.3$ & 1.32 & 1.27 & 1.21 \\
\hline GSCL & $22 q 11.21$ & 1.53 & 1.23 & 2.04 \\
\hline HRAS & $11 \mathrm{p} 15.5$ & 2.57 & 1.70 & 3.09 \\
\hline INS & $11 \mathrm{p}$ tel & 1.30 & 3.00 & 2.68 \\
\hline MYCN (N-myc) & $2 \mathrm{p} 24.1$ & 1.21 & 1.34 & 1.41 \\
\hline PTGS2 (COX2) & 1q31.1 & 1.58 & 1.28 & 2.33 \\
\hline RB1 & $13 q 14$ & 1.39 & 1.30 & 1.61 \\
\hline SNRPN & $15 q 12$ & 1.21 & 1.53 & 2.01 \\
\hline SRY & Yp11.3 & 1.35 & 1.29 & 1.86 \\
\hline D21S378 & $21 q 11.2$ & 1.35 & 1.18 & 1.26 \\
\hline D5S23 & $5 \mathrm{p} 15.2$ & 1.30 & 1.32 & 2.05 \\
\hline D6S434 & $6 q 16.3$ & 1.33 & 1.05 & 1.84 \\
\hline D2S447 & $2 q$ tel & 1.88 & 2.54 & 1.06 \\
\hline ABL1 & $9 q 34.1$ & 1.33 & 1.02 & 1.42 \\
\hline D3S1274, ROBO1 & $3 p 12-3 p 13$ & 1.33 & 0.90 & 1.61 \\
\hline DCC & $18 \mathrm{q} 21.3$ & 1.83 & 1.10 & 2.84 \\
\hline $\mathrm{BRCA} 2$ & $13 q 12-q 13$ & 1.23 & 0.95 & 1.24 \\
\hline CDK6 & $7 q 21-22$ & 1.30 & 0.71 & 1.54 \\
\hline E2F5 & $8 \mathrm{p} 22-\mathrm{q} 21.3$ & 2.09 & 1.17 & 2.47 \\
\hline PIM1 & $6 \mathrm{p} 21.2$ & 1.62 & 1.09 & 1.85 \\
\hline CSF1R & $5 q 33-35$ & 1.08 & 1.31 & 1.25 \\
\hline SGC34236 & $2 q 13$ & 1.11 & 1.44 & 2.09 \\
\hline WHSC1 & $4 \mathrm{p} 16.3$ & 1.10 & 1.23 & 1.54 \\
\hline PDGFB (SIS) & $22 q 13.1$ & 0.56 & 1.63 & 2.02 \\
\hline OCRL1 & $\mathrm{Xq} 25$ & 0.85 & 1.24 & 1.30 \\
\hline KAL & $\mathrm{Xp} 22.3$ & 1.19 & 1.21 & 1.96 \\
\hline IGH (SHGC-36156) & $14 \mathrm{q}$ tel & 1.14 & 0.82 & 1.35 \\
\hline D6S268 & 6q16.3-q21 & 1.18 & 1.00 & 1.47 \\
\hline D13S319 & $13 q 14.2$ & 1.08 & 1.10 & 2.09 \\
\hline
\end{tabular}

Table II. Continued.

\begin{tabular}{|c|c|c|c|c|}
\hline \multicolumn{5}{|l|}{ B, Deletions } \\
\hline $\begin{array}{l}\text { Genes and } \\
\text { regions }\end{array}$ & $\begin{array}{c}\text { Chromosomal } \\
\text { locus }\end{array}$ & 1 & 2 & 3 \\
\hline EGR2 & $10 \mathrm{q} 21.3$ & 0.80 & 0.62 & 0.68 \\
\hline ESR1 & $6 \mathrm{q} 25.1$ & 0.73 & 0.81 & 0.66 \\
\hline EST CDY16 & $\mathrm{X} / \mathrm{Yq}$ tel & 0.77 & 0.77 & 0.62 \\
\hline MLL & $11 \mathrm{q} 23$ & 0.82 & 0.80 & 0.81 \\
\hline PIK3CA & $3 q 26.3$ & 0.81 & 0.69 & 0.67 \\
\hline MAP2K5 & $15 \mathrm{q} 23$ & 0.67 & 0.93 & 0.66 \\
\hline RAF1 & $3 \mathrm{p} 25$ & 0.81 & 1.02 & 0.75 \\
\hline MYB & $6 q 22-q 23$ & 0.83 & 0.58 & 0.68 \\
\hline ABCB1 (MDR1) & $7 \mathrm{q} 21.1$ & 1.00 & 0.55 & 0.72 \\
\hline CDKN 2A (p16) & $9 \mathrm{p} 21$ & 0.85 & 0.76 & 0.66 \\
\hline CYP 24 & $20 \mathrm{q} 13.2$ & 0.91 & 0.66 & 0.73 \\
\hline D1S2465 & $1 \mathrm{p} 12$ & 0.92 & 0.65 & 0.61 \\
\hline ITGA4 & $2 q 31-q 32$ & 0.91 & 0.62 & 0.76 \\
\hline SHGC-182 & 1q tel & 1.17 & 0.57 & 0.69 \\
\hline TGFB2 & $1 \mathrm{q} 41$ & 0.90 & 0.79 & 0.73 \\
\hline stSG42796 & $19 \mathrm{p}$ tel & 1.13 & 0.57 & 0.73 \\
\hline TERC & $3 q 26$ & 1.05 & 0.65 & 0.75 \\
\hline RASSF1 & $3 \mathrm{p} 21.3$ & 0.78 & 1.49 & 0.96 \\
\hline IGH & $14 \mathrm{q}$ tel-2 & 1.14 & 0.82 & 1.35 \\
\hline DAB2 & $5 \mathrm{p} 13$ & 0.98 & 0.67 & 0.91 \\
\hline KAI1 & $11 \mathrm{p} 11.2$ & 0.94 & 0.83 & 0.67 \\
\hline BCL2 3' & $18 \mathrm{q} 21.3$ & 0.92 & 0.83 & 0.65 \\
\hline D19S238E & $19 q$ tel & 1.00 & 0.93 & 0.77 \\
\hline LPL & $8 \mathrm{p} 22$ & 0.94 & 0.89 & 0.65 \\
\hline TCL1A & $14 \mathrm{q} 32.1$ & 0.88 & 0.99 & 0.81 \\
\hline ABCC1 (MRP1) & $16 \mathrm{p} 13.1$ & 0.88 & 0.95 & 0.72 \\
\hline D6S311 & 6p23-24 & 0.94 & 0.87 & 0.81 \\
\hline TK1 & $17 \mathrm{q} 23.2-\mathrm{q} 25.3$ & 0.98 & 0.90 & 0.75 \\
\hline
\end{tabular}

ACGHs were performed using DNA from whole formalin-fixed, paraffinembedded lymph node biopsies of individual classic HL patients who developed secondary AML at diagnosis as test and pooled DNAs from 8 reactive lymph nodes as reference. Panels $\mathrm{A}$ and $\mathrm{B}$ show in sequence amplifications and deletions seen in 3,2 or only $1 \mathrm{HL}$ patient. Bold characters distinguish genes and regions shared by at least 2 out of 3 classic HL patients comparable for histological type, clinical stage and follow-up who did not develop any secondary malignancy (see Table IIIA and B).

from 3 classic HL patients who did not develop any secondary cancer (comparable for histological type, clinical stage and follow-up) as tests and pooled DNAs from 8 reactive lymph node biopsies as reference (Table IIIA and B). They encompass genes involved in genomic stability and concerning, in particular, telomeric regions (16PTEL03, 20QTEL14, 6QTEL54, 8M16/SP6, INS, EST CDY16, stSG42796), growth factor and hormone receptors (EGR1, ERBB2, FES, EGR2, ESR1), regulators of cell cycle progression (RB1, CDKN2A), apoptosis [PTGS2 (COX2)] and chromatin epigenetic structure (MLL), and signal transduction signals (HRAS, MYCN, MYB, PIK3CA, MAPK2K5). In conclusion, our results suggest a 
Table III. Genomic imbalances in HL patients who did not develop any secondary malignancy relative to normal controls.

\begin{tabular}{|c|c|c|c|}
\hline \multicolumn{4}{|l|}{ A, Amplifications } \\
\hline Genes and regions & 4 & 5 & 6 \\
\hline 16PTEL03 & 1.14 & 1.35 & 1.01 \\
\hline 20QTEL14 & 1.12 & 3.08 & 1.87 \\
\hline 6QTEL54 & 1.09 & 3.55 & 1.87 \\
\hline 7QTEL20 & 1.04 & 1.70 & 1.03 \\
\hline 8M16/SP6 & 1.11 & 3.46 & 1.97 \\
\hline ARHGAP8 & 0.95 & 1.21 & 0.96 \\
\hline ATM & 1.16 & 2.81 & 1.06 \\
\hline $\mathrm{CDC} 2 \mathrm{~L} 1$ (p58) & 1.11 & 1.62 & 1.15 \\
\hline D10S167 & 1.09 & 1.73 & 1.24 \\
\hline D13S25 & 1.45 & 1.61 & 2.77 \\
\hline D17S1670 & 0.92 & 2.20 & 1.36 \\
\hline EGR1 & 1.69 & 2.64 & 1.23 \\
\hline ELN & 1.49 & 2.51 & 1.26 \\
\hline ERBB2 (HER-2) & 1.08 & 2.29 & 1.54 \\
\hline FES & 1.64 & 1.78 & 2.32 \\
\hline GLI & 1.13 & 1.40 & 1.24 \\
\hline GSCL & 1.08 & 2.56 & 1.45 \\
\hline HRAS & 1.35 & 4.43 & 2.75 \\
\hline INS & 1.34 & 3.87 & 2.35 \\
\hline MYCN (N-myc) & 1.29 & 1.50 & 1.75 \\
\hline PTGS2 (COX2) & 1.03 & 2.61 & 1.82 \\
\hline RB1 & 1.00 & 2.15 & 1.61 \\
\hline SNRPN & 1.20 & 2.20 & 1.43 \\
\hline SRY & 1.07 & 1.95 & 1.33 \\
\hline D21S378 & 1.41 & 1.73 & 2.59 \\
\hline D5S23 & 1.07 & 3.14 & 1.40 \\
\hline D6S434 & 1.29 & 2.22 & 2.23 \\
\hline D2S447 & 4.08 & 0.96 & 2.74 \\
\hline ABL1 & 0.98 & 1.75 & 1.03 \\
\hline D3S1274, ROBO1 & 1.01 & 2.54 & 1.31 \\
\hline DCC & - & 4.45 & 1.63 \\
\hline BRCA2 & 0.84 & 1.51 & 0.92 \\
\hline CDK6 & 0.92 & 1.86 & 1.29 \\
\hline E2F5 & 1.09 & 4.08 & 2.29 \\
\hline PIM1 & 1.17 & 2.95 & 1.66 \\
\hline CSF1R & 1.38 & 1.54 & 1.77 \\
\hline SGC34236 & 1.21 & 2.43 & 1.31 \\
\hline WHSC1 & 1.25 & 1.53 & 2.17 \\
\hline PDGFB (SIS) & 1.24 & 1.56 & 1.13 \\
\hline OCRL1 & 1.16 & 0.95 & 1.36 \\
\hline KAL & 0.89 & 2.46 & 1.50 \\
\hline IGH (SHGC-36156) & 0.84 & 1.93 & 1.23 \\
\hline D6S268 & 1.10 & 1.79 & 1.28 \\
\hline D13S319 & 0.90 & 2.42 & 1.28 \\
\hline AFM217YD10 & 1.36 & 1.66 & 1.88 \\
\hline FGR(SRC2) & 1.44 & 1.29 & 2.20 \\
\hline GATA3 & 1.09 & 1.88 & 1.36 \\
\hline TOP1 & 1.61 & 1.98 & 2.51 \\
\hline WT1 & 1.80 & 1.91 & 2.52 \\
\hline DRIM, ARL1 & 1.21 & 1.28 & 1.31 \\
\hline TBR1 & 1.35 & 1.24 & 1.58 \\
\hline
\end{tabular}

Table III. Continued.

B, Deletions

\begin{tabular}{|c|c|c|c|}
\hline Genes and regions & 4 & 5 & 6 \\
\hline EGR2 & 0.78 & 0.66 & 0.57 \\
\hline ESR1 & 0.78 & 0.72 & 0.64 \\
\hline EST CDY16 & 0.69 & 0.70 & 0.53 \\
\hline MLL & 0.79 & 0.78 & 0.41 \\
\hline PIK3CA & 0.66 & 0.94 & 0.38 \\
\hline MAP2K5 & 0.76 & 0.68 & 0.55 \\
\hline RAF1 & 1.07 & 0.78 & 0.86 \\
\hline MYB & 0.84 & 0.67 & 0.53 \\
\hline ABCB1 (MDR1) & 0.82 & 0.63 & 0.46 \\
\hline CDKN 2A (p16) & 0.71 & 0.67 & 0.55 \\
\hline CYP 24 & 0.80 & 0.66 & 0.46 \\
\hline D1S2465 & 0.72 & 0.61 & 0.53 \\
\hline ITGA4 & 0.81 & 0.74 & 0.73 \\
\hline SHGC-182 & 0.68 & 0.60 & 0.42 \\
\hline TGFB2 & 0.81 & 0.75 & 0.63 \\
\hline stSG42796 & 0.83 & 0.90 & 0.76 \\
\hline TERC & 0.99 & 0.72 & 0.65 \\
\hline RASSF1 & 1.21 & 0.50 & 0.94 \\
\hline IGH & 0.86 & 0.63 & 0.56 \\
\hline DAB2 & 0.87 & 0.85 & 0.62 \\
\hline KAI1 & 0.90 & 0.79 & 0.49 \\
\hline BCL2 3' & 0.79 & 0.62 & 0.47 \\
\hline D19S238E & 1.01 & 0.69 & 0.79 \\
\hline LPL & 0.77 & 0.68 & 0.64 \\
\hline TCL1A & 0.97 & 0.70 & 0.83 \\
\hline ABCC1 (MRP1) & 1.12 & 0.70 & 0.96 \\
\hline D6S311 & 1.08 & 0.78 & 1.02 \\
\hline TK1 & 0.91 & 0.85 & 0.59 \\
\hline
\end{tabular}

ACGH were performed using DNA from whole formalin-fixed, paraffinembedded lymph node biopsies of individual classic HL patients at diagnosis (comparable to those who developed AML for histological type, clinical stage and follow-up) as test and pooled DNAs from 8 reactive lymph nodes as reference. Bold characters distinguish genes and regions shared by at least 2 out of 3 of them.

wide individual variability in the DNA sequence copy number associated with classic HL likely independent from further evolution of the disease.

To distinguish genomic imbalances eventually associated with the development of secondary AML we set aCGH using individual DNAs from lymph node biopsies of classic HL patients who developed AML as tests and pooled DNAs from lymph nodes of $3 \mathrm{HL}$ patients (comparable for histological type, clinical stage and follow-up) who did not develop any secondary malignancy as reference. ACGH results underscored a high degree of variability in genomic imbalances associated with AML development concerning all genes and regions whose copy number relative to normal controls was altered (Table VI). The number of amplifications and deletions in patient 3 (the only one exhibiting complex karyotypic abnormalities in leukemic progenitors) largely exceeded that 
Table VI. Genomic imbalances in classic HL patients who developed AML relative to HL patients who did not develop any secondary malignancy.

\begin{tabular}{|c|c|c|c|}
\hline Genes and regions & 1 & 2 & 3 \\
\hline 6QTEL54 & 0.81 & 0.88 & 1.41 \\
\hline 7QTEL20 & 0.91 & 1.23 & 1.38 \\
\hline 8M16/SP6 & 0.79 & 0.89 & 1.28 \\
\hline ATM & 0.96 & 0.93 & 1.59 \\
\hline CDC2L1 (p58) & 0.75 & 0.91 & 1.31 \\
\hline D10S167 & 0.87 & 1.02 & 1.34 \\
\hline D17S1670 & 0.81 & 0.99 & 1.21 \\
\hline EGR1 & 0.80 & 0.87 & 1.25 \\
\hline ELN & 0.74 & 0.97 & 1.39 \\
\hline ERBB2 (HER-2) & 0.86 & 1.07 & 1.35 \\
\hline GSCL & 1.01 & 1.04 & 1.44 \\
\hline HRAS & 4.73 & 1.01 & 1.08 \\
\hline INS & 0.85 & 1.28 & 1.35 \\
\hline PTGS2 (COX2) & 1.05 & 0.96 & 1.72 \\
\hline RB1 & 0.93 & 0.95 & 1.47 \\
\hline SNRPN & 0.92 & 0.93 & 1.78 \\
\hline SRY & 0.79 & 0.83 & 1.22 \\
\hline D21S378 & 0.87 & 0.68 & 1.21 \\
\hline D5S23 & 1.08 & 0.99 & 1.64 \\
\hline D3S1274, ROBO1 & 1.00 & 0.87 & 1.53 \\
\hline CDK6 & 1.01 & 0.99 & 1.51 \\
\hline E2F5 & 0.79 & 0.92 & 1.32 \\
\hline PIM1 & 0.89 & 0.94 & 1.36 \\
\hline SGC34236 & 0.81 & 0.96 & 1.37 \\
\hline IGH (SHGC-36156) & 1.04 & 0.92 & 1.21 \\
\hline D6S268 & 0.88 & 0.89 & 1.24 \\
\hline D13S319 & 0.86 & 0.99 & 1.57 \\
\hline
\end{tabular}

B, Deletions

\begin{tabular}{lccc}
\hline Genes and regions & 1 & 2 & 3 \\
\hline EGR2 & 1.28 & $\mathbf{0 . 7 9}$ & $\mathbf{0 . 6 1}$ \\
ESR1 & 1.08 & $\mathbf{0 . 7 6}$ & $\mathbf{0 . 5 8}$ \\
EST CDY16 & 1.31 & $\mathbf{0 . 8 1}$ & $\mathbf{0 . 6 9}$ \\
MAP2K5 & 1.18 & 0.96 & $\mathbf{0 . 6 0}$ \\
CDKN 2A (p16) & 1.26 & $\mathbf{0 . 8 0}$ & 0.84 \\
CYP 24 & 1.32 & 0.94 & $\mathbf{0 . 8 1}$ \\
D1S2465 & 1.14 & 0.86 & $\mathbf{0 . 5 0}$ \\
TGFB2 & 1.05 & 0.96 & $\mathbf{0 . 7 0}$ \\
BCL2 3' & 1.11 & 1.12 & $\mathbf{0 . 8 1}$ \\
LPL & 1.03 & 1.06 & $\mathbf{0 . 8 1}$ \\
ABCC1 (MRP1) & 0.99 & 1.18 & $\mathbf{0 . 7 3}$ \\
D6S311 & 0.89 & 0.88 & $\mathbf{0 . 7 8}$ \\
\hline
\end{tabular}

ACGHs were performed using DNA from formalin-fixed, paraffin-embedded lymph node biopsies of individual HL patients who developed AML as test and pooled DNAs from lymph node biopsies of HL patients who did not develop any secondary malignancy (comparable for clinical feature, histological type and follow-up) as reference. Bold characters distinguish genomic imbalances in individual patients. In all cases those genomic imbalances concern genes and regions whose copy number relative to normal controls was found altered (see Table IIA and B).
Table V. Genomic imbalances shared by all 3 HL patients who developed AML relative to HL patients who did not develop any secondary malignancy.

\begin{tabular}{|c|c|c|c|c|}
\hline \multicolumn{5}{|c|}{ A, Amplifications } \\
\hline $\begin{array}{l}\text { Genes and } \\
\text { regions }\end{array}$ & $\begin{array}{c}\text { Chromosomal } \\
\text { locus }\end{array}$ & 1 & 2 & 3 \\
\hline AFM137XA11 & $9 \mathrm{p} 11.2$ & 1.67 & 1.24 & 1.59 \\
\hline FGFR1 & 8p11.2-p11.1 & 1.22 & 1.29 & 1.30 \\
\hline PPARBP & $17 \mathrm{q} 12$ & 1.37 & 1.38 & 1.21 \\
\hline \multicolumn{5}{|l|}{ B, Deletions } \\
\hline $\begin{array}{l}\text { Genes and } \\
\text { regions }\end{array}$ & $\begin{array}{c}\text { Chromosomal } \\
\text { locus }\end{array}$ & 1 & 2 & 3 \\
\hline AFM217YD10 & $17 q$ tel & 0.65 & 0.72 & 0.66 \\
\hline FGR(SRC2) & 1p36.2-p36.1 & 0.56 & 0.81 & 0.66 \\
\hline GATA3 & $10 \mathrm{p} 15$ & 0.75 & 0.79 & 0.61 \\
\hline TOP1 & 20q12-q13.1 & 0.64 & 0.65 & 0.72 \\
\hline WT1 & $11 \mathrm{p} 13$ & 0.59 & 0.69 & 0.68 \\
\hline
\end{tabular}

seen in patients 1 and 2 (Table I). Genomic imbalances shared by all 3 patients included 3 amplifications, encompassing genes and regions whose copy numbers relative to normal controls were not altered, and 5 deletions, due to the loss of amplification seen in classic HL patients who did not develop secondary leukemia) (Table VA and B).

To confirm aCGH results and distinguish the distribution of either amplified or deleted gene product in different lymph node components we performed immunohistochemistry analysis. The results of immunohistochemistry analyses in lymph node biopsies of patients \#2 and \#6 shown in Fig. 3 are representative of all. A significant reduction of the expression of FGR(SRC2) and TOP1 proteins, apparent in lymph node biopsies from HL patients who developed secondary AML compared to those who did not develop any secondary malignant neoplasia, concerned both the neoplastic (RS cells) and non-neoplastic lymph node components (Fig. 3). Conversely, the expression of WT1 protein was lacking in $\mathrm{RS}$ cells of either patient group and significantly reduced in non-neoplastic components of lymph node biopsies of HL patients who developed secondary AML. Furthermore, FGFR1 overexpression in lymph node biopsies of HL patients who developed AML concerned the non-neoplastic lymph node components. Results relative to PPARBP amplification and GATA3 deletion were impaired by the inadequate antibody specificity.

In conclusion, the number of patients included in our study is too limited to assert that genomic imbalances that we found earn consideration as molecular risk factors for secondary AML in HL. Our results must be confirmed in a larger cohort of HL patients. Moreover, the role of individual genomic imbalances in secondary leukemogenesis process requires further investigation. Previous studies proved that 
Control HL \# $2 \quad$ HL \# 6

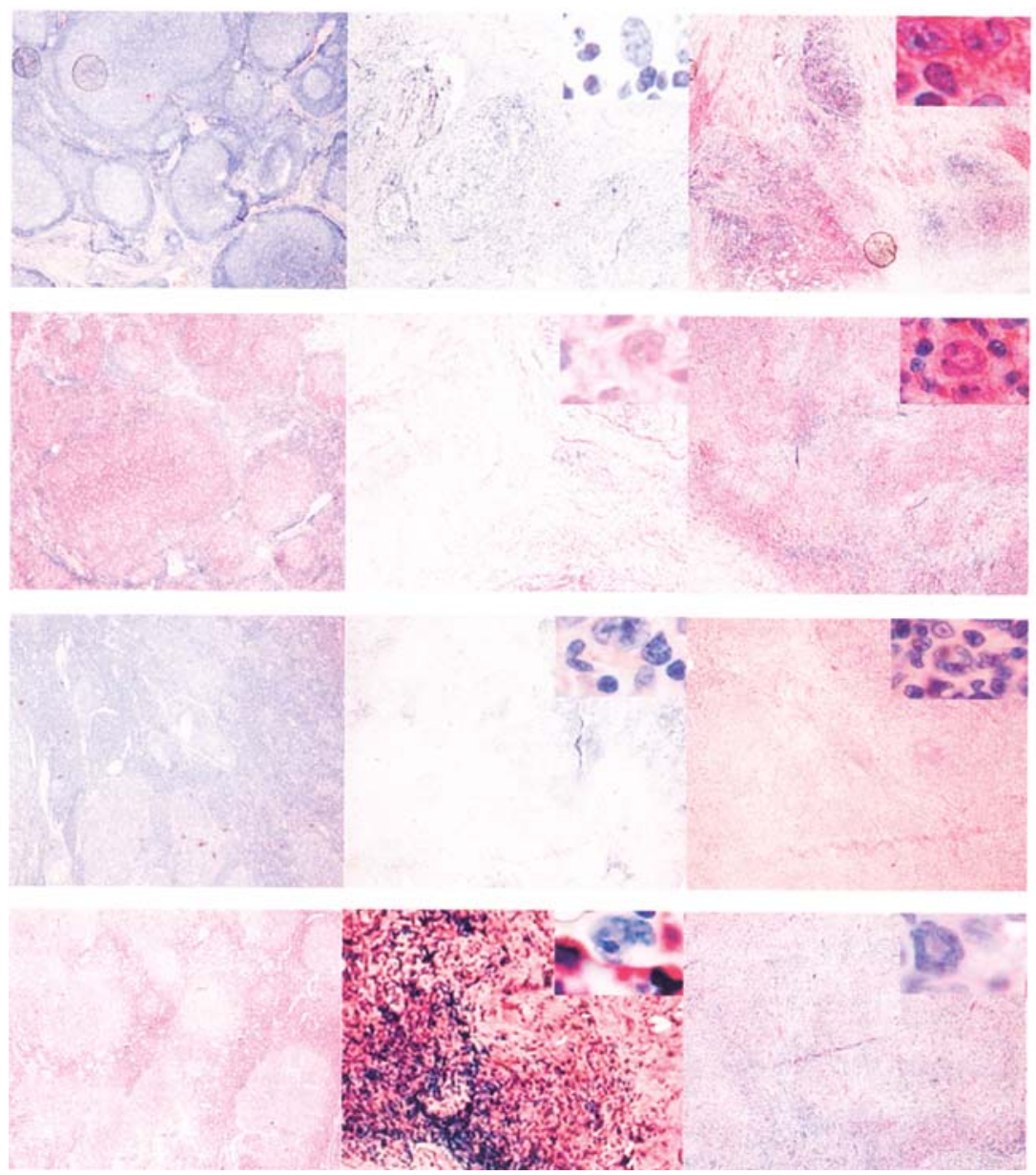

FGR (SRC2)

TOP 1

WT1

FGFR1

Figure 3. Immunohistochemistry of lymph node biopsies from normal persons and classic HL patients. The panels show in sequence FGR(SRC2), TOP1, WT1 and FGFR1 expression in lymph node biopsies from a normal control (reactive lymph node biopsy), one classic HL patient who developed AML (\#2) and one classic HL patient who did not develop any secondary malignancy (\#6). The expression of above mentioned proteins in RS cells are represented in detail in the inserts at the top of the single panels. Results were confirmed in lymph node biopsies of other normal controls and HL patients. Immunohistochemistry analyses were performed on formalin-fixed, paraffin-embedded tissue sections according to the method described in detail in Materials and methods.

WT1 and FGR(SRC2) participate in the immune response against leukemic clones and that TOP1 and FGFR1 intervene in DNA repair $(18,19)$. In particular, TOP1 deletion impairs p53-dependent recombination repair by precluding DNA cleavage in the vicinity of lesions and FGFR1 overexpression targets the centrosome where it activates signalling pathways via tyrosine phosphorylation and allows continuous cell cycle progression $(20,21)$. They might, therefore, concurrently promote the selection of genetic aberrations in an early myeloid compartment leading to the emergence of leukemic progenitors whose recognition and lysis by $\mathrm{CD} 4^{+} \mathrm{T}$ and NKT cells is precluded by WT1 and FGR(SRC2) loss.

\section{Acknowledgements}

The study was supported by the University of Bologna (ex60\% funds and Progetti Pluriennali), Carisbo Foundation and BolognaAIL. G.B. is a Postdoctoral fellow of Dipartimento di Scienze Radiologiche e Istocitopatologiche, University of Bologna-Medical School. M.M. is the recipient of a Postdoctoral grant of the University of Bologna-Medical
School. P.C. is the recipient of a grant from Centro Interdipartimentale di Ricerca sul Cancro 'Giorgio Prodi' of the University of Bologna.

\section{References}

1. Travis LB, Rabkin CS, Brown LM, Allan JM, Alter BP, Ambrosone CB, Begg CB, Caporaso N, Chanock S, DeMichele A, Figg WD, Gospodarowicz MK, Hall EJ, Hisada M, Inskip P, Kleinerman R, Little JB, Malkin D, Ng AK, Offit K, Pui CH, Robison LL, Rothman N, Shields PG, Strong L, Taniguchi T, Tucker MA and Greene MH: Cancer survivorship-genetic susceptibility and second primary cancers: research strategies and recommendations: Review. J Natl Cancer Inst 98: 15-25, 2006.

2. Brauninger A, Schmitz R, Bechtel D, Renne C, Hansmann ML and Kuppers R: Molecular biology of Hodgkin's and Reed/ Sternberg cells in Hodgkin's lymphoma. Int J Cancer 118: 1853-1861, 2006.

3. Re D, Zander T, Dieh V and Wolf J: Genetic instability in Hodgkin's lymphoma. Ann Oncol 13 (Suppl 1): 19-22, 2002.

4. Barrios L, Caballin MR, Miro R, Fuster C, Berrozpe G, Subias A, Batlle $\mathrm{X}$ and Egozcue J: Chromosome abnormalities in peripheral blood lymphocytes from untreated Hodgkin's patients. A possible evidence for chromosome instability. Hum Genet 78: 320-324, 1988. 
5. Erdkamp FL, Schouten HC, Breed WP, Janssen WC, Hoffmann JJ, Schutte B and Blijham GH: DNA aneuploidy in Hodgkin's disease: a multiparameter flow cytometric analysis. Leuk Lymphoma 12: 297-306, 1994.

6. Schlegelberger B, Weber-Matthiesen K, Sterry W, Bartels H, Sonnen R, Maschmeyer G, Feller AC and Grote W: Combined immunophenotyping and karyotyping in peripheral $\mathrm{T}$ cell lymphomas demonstrating different clonal and nonclonal chromosome aberrations in T helper cells. Leuk Lymphoma 15: 113-125, 1994.

7. Jansen MP, Hopman AH, Haesevoets AM, Gennotte IA, Bot FJ, Arends JW, Ramaekers FC and Schouten HC: Chromosomal abnormalities in Hodgkin's disease are not restricted to Hodgkin/ Reed-Sternberg cells. J Pathol 185: 145-152, 1998.

8. Re D, Starostik P, Massoudi N, Staratschek-Jox A, Dries V, Thomas RK, Diehl V and Wolf J: Allelic losses on chromosome 6q25 in Hodgkin and Reed Sternberg cells. Cancer Res 63: 2606-2609, 2003.

9. Wang SS, Davis S, Cerhan JR, Hartge P, Severson RK, Cozen W, Lan Q, Welch R, Chanock SJ and Rothman N: Polymorphisms in oxidative stress genes and risk for non-Hodgkin lymphoma. Carcinogenesis 27: 1828-1834, 2006.

10. Shen M, Zheng T, Lan Q, Zhang Y, Zahm SH, Wang SS, Holford TR, Leaderer B, Yeager M, Welch R, Kang D, Boyle P, Zhang B, Zou K, Zhu Y, Chanock S and Rothman N: Polymorphisms in DNA repair genes and risk of non-Hodgkin lymphoma among women in Connecticut. Hum Genet 119: 659-668, 2006.

11. Re D, Benenson L, Wickenhauser C, Starostik P, StaratschekJox A, Muller-Hermelink HK, Diehl V and Wolf J: Proficient mismatch repair protein expression in Hodgkin and Reed Sternberg cells. Int J Cancer 97: 205-210, 2002.

12. Pedersen-Bjergaard J: Insights into leukemogenesis from therapy-related leukemia. N Engl J Med 352: 1591-1594, 2005.

13. Kelly KM and Perentesis JP: Polymorphisms of drug metabolizing enzymes and markers of genotoxicity to identify patients with Hodgkin's lymphoma at risk of treatment-related complications. Ann Oncol 13 (Suppl 1): 34-39, 2002.
14. Jawad M, Seedhouse CH, Russell N and Plumb M: Polymorphisms in human homeobox HLX1 and DNA repair RAD51 genes increase the risk of therapy-related acute myeloid leukaemia. Blood 108: 3916-3918, 2006.

15. Hattinger CM, Tarkkanen M, Benini S, Pasello M, Stoico G, Bacchini P, Knuutila S, Scotlandi K, Picci P and Serra M: Genetic analysis of fibrosarcoma of bone, a rare tumour entity closely related to osteosarcoma and malignant fibrous histiocytoma of bone. Eur J Cell Biol 83: 483-491, 2004.

16. Liu MT, Chang YT, Chen SC, Chuang YC, Chen YR, Lin CS and Chen JY: Epstein-Barr virus latent membrane protein 1 represses p53-mediated DNA repair and transcriptional activity. Oncogene 24: 2635-2646, 2005.

17. Sanchez-Aguilera A, Montalban C, de la Cueva P, Sanchez-Verde L, Morente MM, Garcia-Cosio M, Garcia-Larana J, Bellas C, Provencio M, Romagosa V, de Sevilla AF, Menarquez J, Sabin P, Mestre MJ, Mendez M, Fresno MF, Nicolas C, Piris MA, Garcia JF and Spanish Hodgkin Lymphoma Study Group: Tumor microenvironment and mitotic checkpoint are key factors in the outcome of classic Hodgkin lymphoma. Blood 108: 662-668, 2006.

18. Guo Y, Niiya H, Azuma T, Uchida N, Yakushijin Y, Sakay I, Hato T, Takahashi M, Senju S, Nishimura Y and Yasukawa M: Direct recognition and lysis of leukemia cells by WT1-specific $\mathrm{CD}^{+} \mathrm{T}$ lymphocytes in an HLA class II-restricted manner. Blood 106: 1415-1418, 2005.

19. Sasanuma H, Tatsuno A, Hidano S, Ohshima K, Matsuzaki Y, Hayashi K, Lowell CA, Kitamura D and Goitsuka R: Dual function for the adaptor MIST in IFN-gamma production by NK and CD4+NKT cells regulated by the Src kinase Fgr. Blood 107: 3647-3655, 2006.

20. Gurevich P, Aplan DA and Humphries RK: NUP98Topoisomerase I acute myeloid leukemia-associated fusion gene has potent leukemogenic activities independent of an engineered catalytic site mutation. Blood 104: 1127-1136, 2004.

21. Karajannis MA, Vincent L, Direnzo R, Shmelkov SV, Zhang F, Feldman EJ, Zhu Z, Sun H, Kussie P and Rafii S: Activation of FGFR 1beta signaling pathway promotes survival, migration and resistance to chemotherapy in acute myeloid leukemia cells. Leukemia 20: 979-986, 2006. 